\title{
A New Inpainting Method for Highlights Elimination by Colour Morphology
}

\author{
Francisco Ortiz and Fernando Torres \\ Automatics, Robotics and Computer Vision Group Dept. Physics, \\ Systems Engineering and Signal Theory. University of Alicante, \\ P.O. Box 99, 03080 Alicante, Spain \\ \{fortiz, Fernando.torres\} @ua.es
}

\begin{abstract}
In this paper, we present a new application of the mathematical morphology: a single-image approach for the automatic detection and elimination of highlights in colour images. We use a 2D-histogram that allows us to relate the achromatic and saturation signals of a colour image and to identify interior brightness. To eliminate the highlights detected, we use an image-inpainting method, by means of connected vectorial filters of the mathematical morphology. This new filter operates exclusively on bright zones, reducing the high cost of processing the connected filters and avoiding over-simplification. The new method proposed here achieves good results, which are similar to those obtained from other multimedia techniques, yet does not require either costly multiple-view systems or stereo images.
\end{abstract}

\section{Introduction}

In visual systems, images are acquired in work environments in which illumination plays an important role. Sometimes, a bad adjustment of the illumination can introduce highlights (brightness or specular reflectance) into the objects captured by the vision system. Highlights in images have long been disruptive to computer-vision algorithms. The presence of such brightness alters the pattern recognition process because the previous stage of detection of edges in the objects fails: in a morphological watershed, the highlights and specular reflectances are considered as different objects in the environment in which they are located and therefore it is not possible to perfectly detect the objects in the scene.

To effectively eliminate the highlights in captured scenes, we must first identify them. The dichromatic reflection model, proposed by Shafer [1], is one tool that has been used in many methods for detecting specularities. It supposes that the interaction between the light and any dielectric material produces different spectral distributions within the object (specular and diffuse reflectance). The specular reflectance has the same spectral makeup as the incident light, whereas, the diffused component is a product of illumination and surface pigments. Based on this model, Lin et al [2] have developed a system for eliminating specularities in image sequences by means of stereo correspondence. Bajcsy et al [3] use a chromatic space based on polar coordinates that allows the detection of specular and diffuse reflections by means of the 
previous knowledge of the captured scene. Klinker et al [4] employ a pixel-clustering algorithm that has been shown to work well in detecting brightness in images of plastic objects.

Wolff [5], for his part, removes highlights by taking advantage of differences in polarization between diffuse reflections and highlights. These above-mentioned approaches have produced good results but entail requirements that limit their applicability, such as the use of stereo or multiple-view systems, a long processing time, the previous knowledge of the scene, or the assumption of a homogeneous illumination. Furthermore, some techniques merely detect brightness without eliminating it.

In this paper, we explain a new application of the mathematical morphology, an automatic and single-image system for the detection and elimination of brightness in colour images. The organisation of this paper is as follows: In Section 2, we present the extension of the geodesic operations to colour images. Section 3 shows the algorithm used for detecting highlights. The elimination process and our experimental results are presented in Section 4. Finally, our conclusions are outlined in the final section.

\section{Vector Connected Filters in HSV Colour Space}

Morphological filters by reconstruction have the property of suppressing details while preserving the contours of the remaining objects [6,7]. The use of such filters in colour images requires an ordered relationship among the pixels of the image. For the vectorial morphological processing, the HSV colour space with a lexicographical ordering $o_{l e x}=v \rightarrow s \rightarrow h$ [8], will be used.

Once the orders have been defined, the morphological operators for the reconstruction of colour images can be applied. Geodesic dilation is an elementary geodesic operation. Let $\boldsymbol{g}$ denote a marker colour image and $\boldsymbol{f}$ a mask colour image (if $\mathrm{o}_{l e x}(\boldsymbol{g}) \leq \mathrm{o}_{l e x}(\boldsymbol{f})$, then $\left.\boldsymbol{g} \wedge_{v} \boldsymbol{f}=\boldsymbol{g}\right)$. The vectorial geodesic dilation of size 1 of the marker image $g$ with respect to the mask $\boldsymbol{f}$ can therefore be defined as:

$$
\delta_{v f}^{(1)}(g)=\delta_{v}^{(1)}(g) \wedge_{v} f
$$

where $\delta_{v}^{(1)}(g)$ is the vectorial dilation of size 1 of the marker image $g$. This propagation is limited by the colour mask $f$. The vectorial geodesic dilation of size $n$ of a marker colour image $g$ with respect to a mask colour image $f$ is obtained by performing $n$ successive geodesic dilations of $\boldsymbol{g}$ with respect to $f$ :

$$
\delta_{v \boldsymbol{f}}^{(n)}(\boldsymbol{g})=\delta_{v \boldsymbol{f}}^{(1)}\left[\delta_{v \boldsymbol{f}}^{(n-1)}(\mathrm{g})\right]
$$

with $\delta_{v f}^{(0)}(g)=f$.

Geodesic transformations of images always converge after a finite number of iterations. The propagation of the marker image is limited by the mask image. Morphological reconstruction of a mask image is based on this principle. The 
vectorial reconstruction by dilation of a mask colour image $f$ from a marker colour image $\boldsymbol{g}$, (both with $\mathrm{D}_{f}=\mathrm{D}_{g}$ and $o_{l e x}(\boldsymbol{g}) \leq o_{l e x}(\boldsymbol{f})$ ) can be defined as:

$$
R_{v_{f}}(g)=\delta_{v f}^{(n)}(g)
$$

where $n$ is such that $\delta_{v}{ }_{\boldsymbol{f}}^{(n)}(\boldsymbol{g})=\delta_{v}{ }^{(n+1)}(g)$.

\section{Highlight Detection by HSV Colour Space}

It is known that the specularities in the chromatic image have a high value (achromatic signal) and a low saturation in the HSV colour model. Androutsos et al in [9] make a division of the luminance-saturation space (HLS) and they conclude that if the saturation is greater than a $20 \%$ and the luminance is greater than a $75 \%$, the pixels are chromatic, while if the saturation is lower than a $20 \%$ and the luminance is greater than $75 \%$, the pixels are very luminous or highlights. Our criterion is similar and it is based, initially, on the division of the value-saturation space in different homogenous regions that segment the chromatic image.

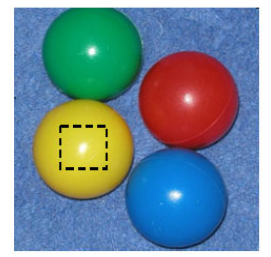

(a)

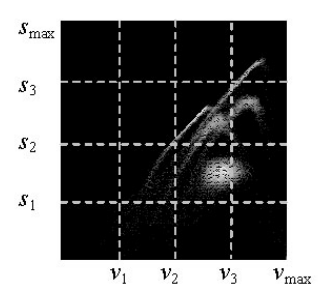

(b)

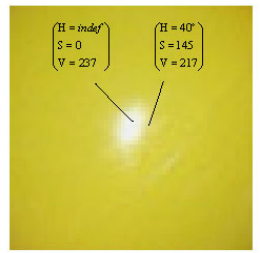

(c)

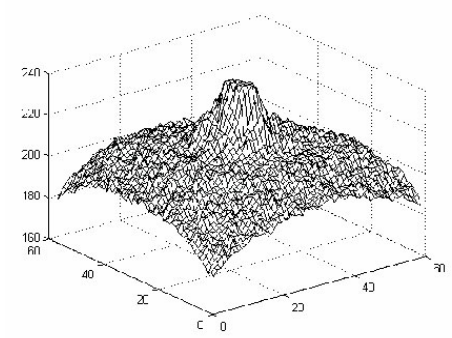

(d)

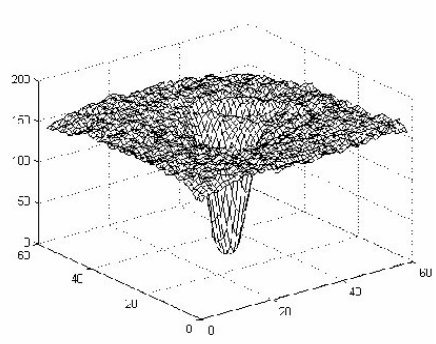

(e)

Fig. 1. Identification of highlights in colour image by co-ordinates of VS diagram. (a) Original colour image. (b) Grouping of co-ordinates in VS diagram, $s_{1}=(1 / 4) s_{\max }, s_{2}=(1 / 2) s_{\max }$, $s_{3}=(3 / 4) s_{\max }, s_{\max }=255, v_{1}=(1 / 4) v_{\max }, v_{2}=(1 / 2) v_{\max }, v_{3}=(3 / 4) v_{\max }, v_{\max }=255$, (c) Highlights in a zone of original colour image. (d) 3D map of value signal in highlighted area. (e) 3D map of saturation signal in highlighted area. 
The hue signal is not used because it is very unstable. The co-ordinates $\left[\left(v_{3^{-}}\right.\right.$$\left.\left.v_{\max }\right),\left(0-s_{1}\right)\right]$ of the VS diagram (Figure 1.b) define the exact limits of the region in which the highlights of a contrasted colour image (Fig.1.a) are present. Different coordinates within this region will identify from the highest or strong specular reflection (central sparkle of brightness) to even soft inter-reflections, which generally represent the transition from specular reflectance to diffuse reflectance. Figure 1.c shows a data transition (in HSV co-ordinates) for a bright area (yellow balloon) of the original image. The great $v$ and smaller $s$ of highlighted area can be seen in Figures 1.d and 1.e.

All the highlights will be located in $\left[\left(v_{3}-v_{\max }\right),\left(0-s_{1}\right)\right]$ region of the VS diagram, but not all of the co-ordinates in that area will correspond to specularities. The achromatic axes zone could be considered to be a highlight. This is partly true, as it only occurs in grey-scale images. In the HSV colour space, if value $v$ decreases, the brightness has a similar surface colour (diffuse reflection) as the objects on which such highlight appears and the saturation $s$ is increased.

Another important aspect to be taken into consideration is that not all of the images will have the same dynamic range and, therefore, the signals of value $v$ and saturation $s$ of highlights will not always correspond to the region $\left[\left(v_{3}-v_{\max }\right),\left(0-s_{1}\right)\right]$ of the VS diagram, previously presented. In addition, what happens in the case of colored light sources (incandescent lamps, A, C)? A new contrast enhancement is the solution.

\subsection{Contrast Enhancement by Colour Morphology}

The previous problems of highlights localization could be solved with a contrast enhancement by histogram equalisation. Nevertheless, the histogram equalisation of the original image is only feasible in the achromatic signal. Furthermore, this operation might well cause an excessive increase in value $v$, an over-saturation and a false detection of brightness.

The best solution is to apply a new vector-morphological contrast enhancement for luminous pixels, which operates in chromatic images by means of a previously established lexicographical order $o_{\text {lex }}=v \rightarrow s \rightarrow h$. Specifically, the vector top-hat operator is added to the original image to enhance bright objects [10]. We denote the colour-morphological contrast enhancement by:

$$
\boldsymbol{f}^{\prime}=\boldsymbol{f}+W T H_{v}(\boldsymbol{f})
$$

where $\boldsymbol{f}^{\prime}$ is the new contrasted colour image and $W T H_{v}(\boldsymbol{f})$ is the vectorial top-hat by opening, which is made with a structuring element of size $t$. The top-hat is defined as follows:

$$
W T H_{v}(f)=f-\gamma_{\mathrm{v}}(\boldsymbol{f})
$$

The contrast enhancement achieved with this new operator is visually very good. Furthermore, the operation expels the highlights to the limits of the RGB cube. In the HSV colour space, the specular reflectance is located in the co-ordinate $v_{\max }$ with minimum saturation. As such, all of the specularities are identified along this value, from $s=0$ to a threshold of $s$, which we shall denominate by $s_{\mathrm{sp}}$, such that $s_{\mathrm{sp}}<s_{1}$. In our investigation, after a process of morphological contrast enhancement, we have 
observed that the $v$ and $s$ signals of the original image have been altered, approaching the axis $v_{\max }$, with the movement flow shown in Figure 2. Such transitions depend on relationship between the sizes of the highlighted area $(b)$ and the structuring element ( $t$ ) employed in the vectorial operation of top-hat. When $b \leq t$, all of the highlights (strong or weak) are located in the $v_{\max }$ line for any kind of image. In all other cases $(b>t)$, the weaker highlights will be located outside of this co-ordinate, as can be seen in the graph. In Figure 3 we show the difference between the contrast enhancement by histogram equalization and the new morphological contrast operator that does not excessively increase the intensity of the image and it is effective in the regions of mild specularities.

The detection of weak specularities stops in all of the images at a threshold of $s$, smaller than $s_{1}$, (concretely, $\left.s=(1 / 4) s_{1}\right)$. We can thus fix the specularities at $\left[v_{\max },(0-\right.$ $\left.s_{\mathrm{sp}}\right)$ ], where $v_{\max }=255$ and $s_{\mathrm{sp}}=(1 / 4) s_{1}$ or 16 . This is similar to the success of detection of specularities presented in [11] with other polar colour space.
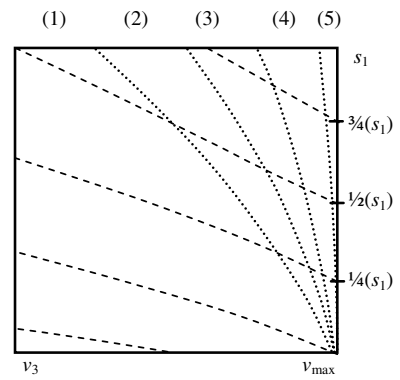

(1) $b>2 t$

(2) $b \leq 2 t$

(3) $b<5 / 3(t)$

(4) $b<4 / 3(t)$

(5) $b \leq t$

Fig. 2. Movement flow for value $v$ and saturation $s$ in the $\left[\left(v_{3}-v_{\max }\right),\left(0-s_{1}\right)\right]$ area of the VS diagram after the new morphological contrast enhancement. All of the highlights are located in the $v_{\max }$ line $\left[v_{\max }, 0\right]$ if highlighted area $(b)$ is smaller than the structuring element $(t)$.

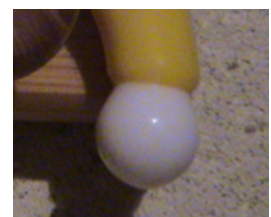

(a)

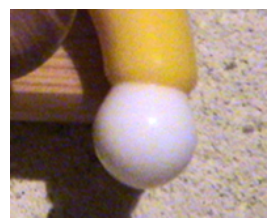

(b)

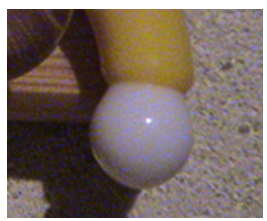

(c)

Fig. 3. Contrast enhancement of color image. (a) Chromatic real scene. (b) Contrast enhancement by histogram equalization. (c) Contrast enhancement by top-hat contrast operator.

\section{Highlight Elimination by Inpainting Process}

Digital inpainting, the technique of reconstructing small damaged portions of an image, has received considerable attention in recent years. This technique has a wide range of applications, including the removal of text, defects or scratches from images. Inpainting methods are based on different strategies. Bertalmio et al [12] pioneered a 
digital image-inpainting algorithm based on partial differential equations (PDEs). Tan et al [13] presented a new single-image highlight removal that incorporates illumination-based constraints into image inpainting. In this paper, we present an inpainting process that is based on colour mathematical morphology, as a traditional inpainting which propagates boundary values.

To eliminate the highlight that was previously detected with the VS diagram, we propose the use of geodesic filters of mathematical morphology. Specifically, a vectorial opening by reconstruction applied exclusively to the specular areas of the image and their surroundings. In this case, a new mask-image $h(x, y)$ represents the pixels of $\boldsymbol{f}$ with which we will be operating. The mask-image $h$ is a dilation of size $(e)$ of the mask of specularities (pixels with $(v, s) \in\left[v_{\max },\left(0-s_{\mathrm{sp}}\right)\right]$ ). Assuming that $\mathrm{D}_{h}=\mathrm{D}_{f}$, each pixel $(x, y)$ has a value of $h(x, y)=\{0,1\}$, where $h(x, y)=1$ in the new areas of interest in the image. The size $e$ of the structural element of the dilation will determine the success of the reconstruction and the final cost of the operations, since this size defines the area to be processed by the filters. In all the cases, the relation $b \leq$ $t \leq e$ must to be satisfied.

In the geodesic filter, $\boldsymbol{f}$ is first eroded. The eroded sets are then used for a reconstruction of the original image. The new filter is then defined, taking into account the fact that, in this case, the operation will not affect all the pixels $(x, y)$, but only those in which $h(x, y)=1$ :

$$
\gamma_{v}^{\left(n^{\prime}\right)}=\left\{\delta_{v \boldsymbol{f}}^{\left(n^{\prime}\right)}\left(\varepsilon_{v}^{(e)}(\boldsymbol{f})\right) \quad \forall \quad \forall \boldsymbol{f}(x, y) \Rightarrow h(x, y)=1\right\}
$$

where $n^{\prime}$ is such that $\delta_{v}{ }_{f}^{\left(n^{\prime}\right)}\left(\varepsilon_{v}^{(e)}(f)\right)=\delta_{v}{ }^{\left(n^{\prime}+1\right)}\left(\varepsilon_{v}^{(e)}(f)\right)$. The vectorial erosion of the opening by reconstruction is also done with a structural element of size $e$. This erosion replaces highlight pixels (high $o_{\text {lex }}$ ) by the surroundings chromatic pixels (low $\left.o_{\text {lex }}\right)$. The vectorial geodesic dilation (iterated until stability is achieved) then reconstructs the colour image without recovering the specularities, as it were demonstrated in [14]. This is the same approach successfully used for the attenuation of the colour objects in medical images [8], the gaussian noise reduction [15] or the filling the holes [16]

The study is carried out on a set of real chromatic images that are quite representative of countless common materials (i.e., plastic, ceramics, fruit, wood, etc.). In Figure 4 we present the results obtained from the application of our geodesic filter in a representative subset of different real scenes. With the new filter, we avoid some of the main inconveniences that arise in geodesic reconstruction: i.e., the high cost of processing due to multiple iterations of the reconstruction and the oversimplification of the image [17].

The size $e$ of the structuring element for morphological operations in highlight elimination depends on: the sizes $b$ and $t$ of the first step of the algorithm, posterior applications and real-time requirements. A low $e(1,2)$ is recommended for visual inspection and a high $e(6,7 \ldots)$ is better for multimedia and image restoration. The new pixel codification of the original highlight is obtained by vectorial inpainting process with the use of our geodesic filter. 


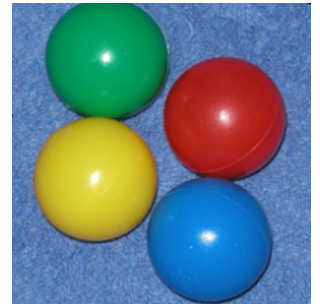

(a)

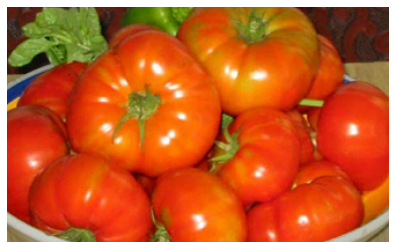

(c)

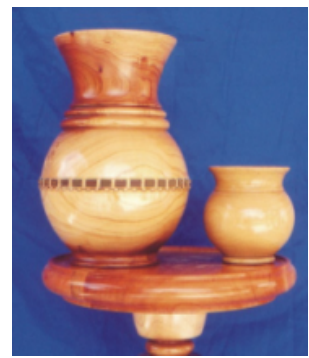

(e)

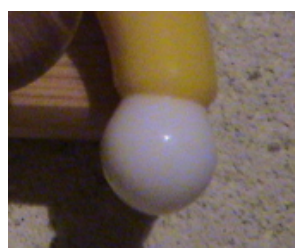

(g)

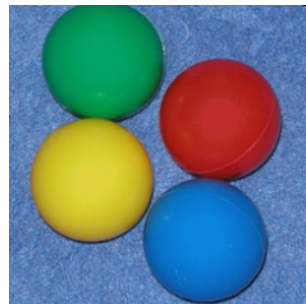

(b)

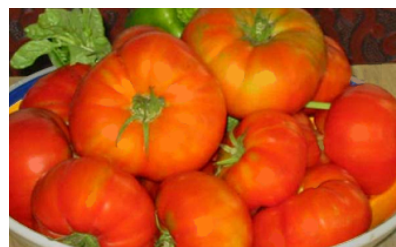

(d)

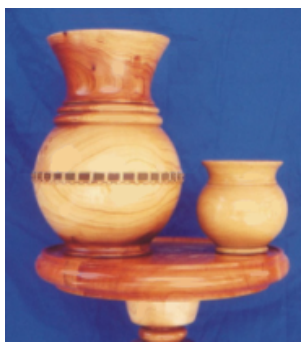

(f)

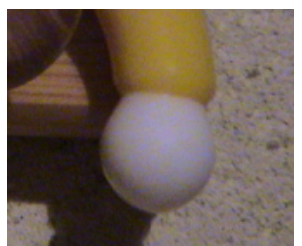

(h)

Fig. 4. Vectorial inpainting by geodesic filter for highlight removal in original colour images. Over-simplification is not present in the results. (a-b) "Balloons", (c-d) "Tomatoes", (e-f) "Vases", (g-h) "Umbrella".

The reduction of the value signal $v$ and the recuperation of saturation $s$ in a detailed area of specular reflectante of "Balloons" image (Figure 1.c) it is quite significant; the indefinite hue for strong highlight also disappears, as can be seen in Figure 5.

Our new method achieves a reduction in total time cost, for all of the images, of between $50 \%$ and $70 \%$, with respect to a global filter. The cost of our inpainting is linear in relation to the size of the structuring element $(e)$ and iterations $(n$ ') of the morphological operations.

In comparison to other non-morphological inpainting methods, and specifically the one presented by Bertalmio et al [12], our method achieves similar visual results in a 


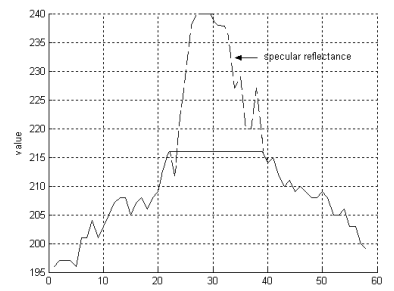

(a)

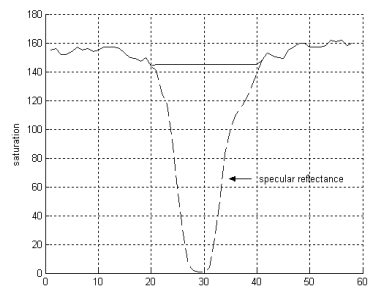

(b)

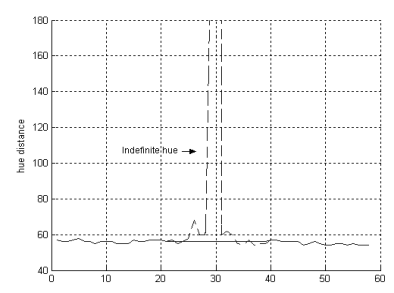

(c)

Fig. 5. 2D-maps of inpainting for removing the specular reflectance in highlight area of "Balloons", $(e=7, t=7, b=5)$. Substitution of: value signal (a), saturation signal (b) and hue (c).

shorter CPU time ( 1 or 2 seconds versus a few minutes for a relatively small inpainting area). An illumination-constrained method presented by Tan et al [13] can lead a more accurate results in inpainting. In addition, the surface textures obscured by highlights are better recovered. Nevertheless, this method requires significant computation.

It should also be noted that our algorithm eliminates the highlight area, and it detects the highlight as well. As in the case of many inpainting methods, the only limitation to this technique is the reconstruction of highly textured areas.

\section{Conclusions}

In this paper, we have presented a new method for the elimination of highlights in colour images for different applications, such as visual inspection, multimedia or restoration.

The use of a new connected vectorial filter allows us to eliminate the specular reflectance previously detected by means an inpainting process. The inpainting is made by an extension of the geodesic transformations of the mathematical morphology to colour images in HSV colour space. The possibility of eliminating highlights in colour images without causing over-simplification has been demonstrated. In addition, the elimination of brightness has been achieved within a very short processing time with respect to a global geodesic reconstruction or other inpainting techniques.

Based on the success shown by these results, we are now working on an improvement of our method for eliminating specularities in real-time environments: we work in multi-processor configurations for colour geodesic operations in order to reduce the processing time required for these operations as much as possible.

\section{References}

1. Shafer, S.A.: Using color to separate reflection components. Color Research Appl. Vol. 10 (1985) 210-218

2. Lin, S. Li, Y., Kang, S., Tong, X., Shum, H.: Diffuse-Specular Separation and Depth Recovery from Image Sequences. Lecture Notes in Computer Science, Springer-Verlag. Vol. 2352 (2002) 
3. Bajcsy, R., Lee, S., Leonardis, A.: Detection of diffuse and specular interface reflections and inter-reflections by color image segmentation. International Journal on Computer Vision. Vol. 17 (1996) 241-271

4. Klinker, G., Shafer, S.A., kanade, T.: Image segmentation and reflection analysis through color. In: Proc. SPIE. Vol. 937 (1988) 229-244

5. Wolff, L.: Using polarization to separate refelction components. In: Proc. IEEE Computer Vision and Pattern Recognition (1989) 363-369

6. Vicent, L.: Morphological Grayscale Reconstruction in Image Analysis: Applications and Efficient Algoritms. IEEE Transactions on Image Processing. Vol. 2. (1993) 176-201

7. Crespo, J. Serra, J., Schafer, R.: Theoretical aspects of morphological filters by reconstruction. Signal Processing. Vol. 47 (1995) 201-225

8. Ortiz, F., Torres, F., De Juan, E., Cuenca, N.: Colour mathematical morphology for neural image analysis. Journal of Real Time Imaging. Vol. 8, i.6 (2002) 455-465

9. Androutsos, D., Plataniotis, K., Venetsanopoulos, A.: A novel vector-based approach to color image retrieval using a vector angular-based distance measure. Computer Vision and Image Understanding, Vol. 75 (1999)

10. Soille, P. A note on morphological contrast enhancement. Technical Report RT-PS-001. École des Mines d'Alès-EERIE (1997)

11. Torres, F., Angulo, J., Ortiz, F.: Automatic detection of specular reflectance in colour images using the MS diagram. Lecture Notes in Computer Science, Springer-Verlag. Vol. 2756 (2003) 132-139

12. Bertalmio, M., Sapiro, G., Caselles V., Ballester, C.: Image Inpainting. In: International Conference on Computer Graphics and Interactive Techniques (2000) 417-424

13. Tan, P., Lin, S., Quon, L., Shum, H.Y.: Highlight removal by illumination-constrained inpainting. In: Proc. of IEEE International Conference on Computer Vision (2003)

14. Ortiz, F., Torres, F.: Vectorial morphological reconstruction for brightness elimination in colour images. Journal of Real Time Imaging. Vol. 8, i.6 (2004) 379-387

15. Ortiz F., Torres F., Gil P.: Gaussian noise elimination in colour images by vectorconnected filters. In: Proc. IEEE 17th International Conference on Pattern Recognition. Vol. 4 (2004) 807-811

16. Soille, P., Gratin, C.: An efficient algorithm for drainage networks extraction on DEMs. In: Journal of Visual Communication and Image Representation. Vol. 5, i.2 (1994) 181-189

17. Ortiz, F.: Procesamiento morfológico de imágenes en color. Aplicación a la reconstrucción geodésica. PhD Thesis, University of Alicante (2002) 